\title{
SEPIAPTERIN REDUCTASE DEFICIENCY: MIMIC OF CEREBRAL PALSY
}

Researchers at University of California at San Diego, and 22 other US national and international centers studied the clinical, biochemical, and molecular findings in a cohort of 38 patients with sepiapterin reductase deficiency (SRD). The phenotype and treatment response were assessed by chart and literature review. Average age of onset is 7 months, and delay to diagnosis is 9.1 years. Symptoms in infancy or childhood include motor and language delays, axial hypotonia, dystonia, weakness, oculogyric crises, with diurnal fluctuation, benefitted by sleep. Cerebral palsy is a common misdiagnosis. CSF showed low 5-hydroxyindoleacetic acid and homovanillic acid and elevated biopterin and sepiapterin. Diagnosis is confirmed by mutation analysis and/or enzyme activity in cultured fibroblasts. All patients treated with 1-dopa in combination with carbidopa showed dramatic improvement. Early diagnosis and treatment are recommended. (Friedman J, Roze E, Abdenur JE, et al. Sepiapterin reductase deficiency: a treatable mimic of cerebral palsy. Ann Neurol 2012 April;71:520-530). (Respond: Dr Jennifer Friedman, Rady Children's Hospital, San Diego, 8010 Frost Street, Suite 400, San Diego, CA 92123. E-mail: jrfriedman@rchsd.org).

COMMENT. A diagnosis of SRD should be considered in patients with developmental delay, dystonia, axial hypotonia and atypical presumed cerebral palsy.

\section{NEONATAL HYPOGLYCEMIA, LACTIC ACIDOSIS, AND PYRIDOXINE-DEPENDENT EPILEPSY}

Researchers at the University of Toronto, Canada report the case of a 13-monthold girl with neonatal hypoglycemia, lactic acidosis, and bilateral symmetrical temporal lobe hemorrhages and thalamic changes on cranial MRI. She developed multifocal and myoclonic seizures refractory to multiple antiepileptic drugs that responded to pyridoxine. A diagnosis of a-aminoadipic semialdehyde dehydrogenase deficiency was confirmed by elevated urinary a-aminoadipic semialdehyde excretion, and a novel missense mutation in the $A L D H 7 A 1$ gene. Seizures were controlled by pyridoxine alone since 1.5 months of age. At 13 months, she has motor delay and central hypotonia but normal language and social development. (Mercimek-Mahmutoglu S, Horvath GA, Coulter-Mackie M, et al. Profound neonatal hypoglycemia and lactic acidosis caused by pyridoxine-dependent epilepsy. Pediatrics 2012 May;129:e1368-e1372).

COMMENT. Pyridoxine-dependent epilepsy is reported with mutations in the $A L D H 7 A 1$ gene that encodes antiquitin. The clinical spectrum of antiquitin deficiency includes ventriculomegaly detected on ultrasound, abnormal fetal movements, a multisystem neonatal disorder, and seizures and autistic features. Brain abnormalities and hypoglycemia that may coexist make diagnosis difficult, and tests for antiquitin deficiency and a clinical trial of pyridoxine are recommended when neonatal seizures are refractory to anticonvulsants. (Mills PB et al. Brain 2010 Jul;133(Pt7):2148-2159). 